\title{
GREEN SYNTHESIS OF PLANT-MEDIATED METAL NANOPARTICLES: THE ROLE OF POLYPHENOLS
}

\author{
LATIF MS, ABBAS S, KORMIN F*, MUSTAFA MK \\ Department of Technology and Natural Resources, Faculty of Applied Sciences and Technology, Universiti Tun Hussein Onn Malaysia \\ Kampus Pagoh, KM1, Jalan Panchor, 84000 Muar, Johor, Malaysia. Email: faridahk@uthm.edu.my
}

Received: 23 March 2019, Revised and Accepted: 04 June 2019

\begin{abstract}
The use of metal nanoparticles (MNPs) in various fields is increasing day-by-day leading to a genuine concern about the issues related to their environmental and biological safety. The major approaches for the synthesis of NPs include physical and chemical methods which are expensive and hazardous to health in addition to being toxic to the environment. This review highlights the potential of plant extracts to carry out the synthesis of MNPs with a special emphasis on the role of flavonoids in nanosynthesis. This green and clean approach have been actively utilized in recent years as an alternative to conventional hazardous approaches. It has proved as cost-effective, non-toxic, less time and labor consuming, efficient, and ecofriendly method for the synthesis of MNPs with specific biological actions. This review also focuses on the role of polyphenols, including the flavonoids as bioreductants of metal salts for the synthesis of NPs along with their biomedical applications. Various examples of the MNPs, along with their biological actions, have also been summarized.
\end{abstract}

Keywords: Green synthesis, Metal nanoparticles, Polyphenols, Flavonoids, Plant extract.

(C) 2019 The Authors. Published by Innovare Academic Sciences Pvt Ltd. This is an open access article under the CC BY license (http://creativecommons. org/licenses/by/4. 0/) DOI: http://dx.doi.org/10.22159/ajpcr.2019.v12i7.33211

\section{INTRODUCTION}

The synthesis of nanoparticles (NPs) can be performed using different methods, including physical, chemical, and biological techniques [1]. The NP synthesis by conventional physical and chemical techniques carries the risk of toxicity and environmental pollution as they release toxic by-products, which are potentially hazardous to the environment [2]. In addition to it, the NPs synthesized by such hazardous methods are unfit for the medical field due to the health-related concerns, particularly in clinical applications [3].

Although the conventional methods are suitable for the synthesis of large quantities of particles, in a lesser period of time, with defined sizes and shapes, these techniques have the drawbacks of being complicated, costly, inefficient, and out fashioned. The recent years have witnessed a growing interest in the nanosynthesis of environment-friendly particles without involving the production of toxic by-products as part of the synthesis process [4-6].

This task is achievable only through adopting environment-friendly synthesis procedures using biotechnology tools of biological nature that is described as safe and environmentally benign for nanosynthesis as an alternative to conventional physical and chemical methods $[7,8]$. This concept has led to the approach of green technology or green nanobiotechnology. In general, the nanosynthesis procedures involving biological routes such as those which are based on microorganisms (viruses, bacteria, fungi, and algae), plants, plant extracts, or their by-products, for example, proteins, lipids, alkaloids, and flavonoids by applying different biotechnology tools and techniques $[9,10]$. A graphical summary of plant-based NP synthesis is shown in Fig. 1.

The superiority of NPs synthesized by green technology to those produced by conventional methods is quite evident due to several features. For instance, green technology employs the use of costeffective chemicals, less energy, and produces eco-friendly products, and by-products. The nanobiotechnology is more advantageous over other conventional procedures due to the fact the more components are available by the biological system for the synthesis of NPs $[11,12]$.
By virtue of the rich biodiversity of biological systems, it is now possible to synthesize the bionanomaterials which are environment-friendly and have the potential to use in a variety of medical applications. Due to the synthesis of environment-friendly chemical products and by-products, the 12 principles of green chemistry are now considered as a reference guide in related research around the world [13]. Consequently, the green nanobiotechnology has now become a promising alternative route for the synthesis of biocompatible and stable NPs [14,15]. In context to the importance of polyphenols including flavonoids of plant extracts in mediating the synthesis of metal NPs (MNPs), this review attempts to highlight and summarize the role of polyphenols in the synthesis of MNPs as described in recent literature.

Biosynthesis of NPs uses a bottom-up approach in which synthesis is performed by the application of reducing and stabilizing agents [16]. There are three main factors which are described for the biosynthesis of NPs based on a biological system: The choice of solvent medium, the choice of an eco-friendly and environmentally benign reducing agent, and the choice of a nontoxic material as a capping agent to stabilize the synthesized NPs [6].

\section{BIOLOGICAL NANOSYNTHESIS AND ITS APPLICATIONS}

In contrast to the physical and chemical methods of nanosynthesis, the biological nanosynthesis relies on the use of microorganisms (bacteria and fungi), enzymes, and plants to produce MNPs [17] (Table 1)

There are numerous examples of a variety of applications of the MNPs in the fields of biomedicine, physicochemistry, agriculture, and environment $[9,18]$, as shown in Fig. 2.

\section{PLANT-BASED NANOMATERIALS}

The plant resources based green synthesis of a number of MNPs including copper $(\mathrm{Cu})$, gold $(\mathrm{Au})$, nickel $(\mathrm{Ni})$, platinum $(\mathrm{Pt})$, titanium (Ti), selenium (Se), silver (Ag), and zinc NPs have already been reported $[19,20]$. The plant-based MNPs have been shown to possess various activities such as antimicrobial, anticancer, antidiabetic, antiinflammatory, antioxidant, and immunomodulatory [21-24]. In recent 


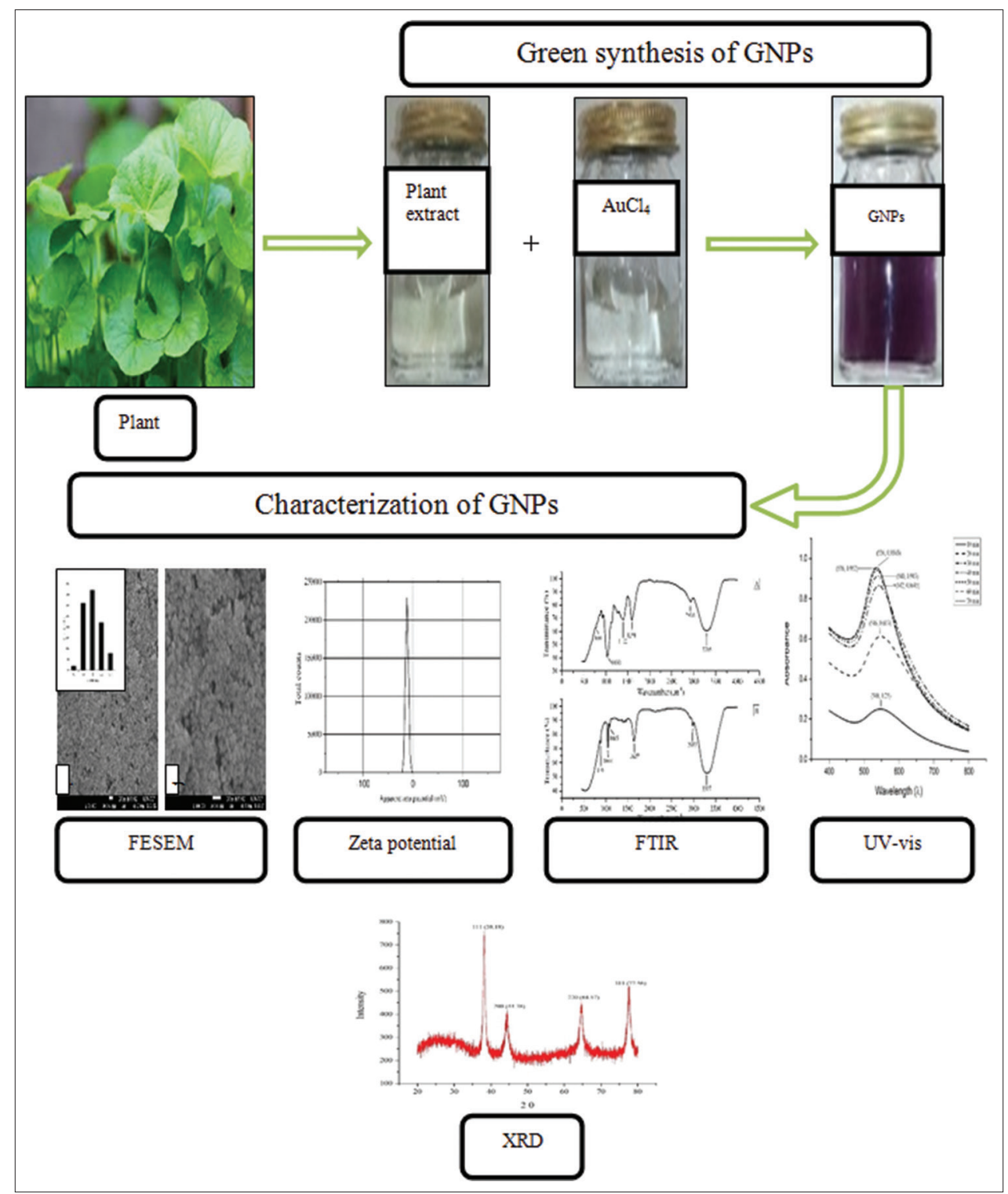

Fig. 1: Green synthesis and characterization of nanoparticles using plants

Table 1: Methods of nanoparticle synthesis

\begin{tabular}{lll}
\hline Physical & Chemical & Green/biological \\
\hline Mechanical methods & Coprecipitation & Plant extracts-mediated \\
Vapor deposition & Sol-gel & Microbial culture-mediated \\
Sputter deposition & Microemulsions & Agricultural waste-mediated \\
Electric deposition & - & Enzymes-mediated \\
Ion beam method & - & - \\
\hline
\end{tabular}

reviews, the MNPs synthesis using various plant extracts has been reported for cobalt, copper, gold, magnetite, platinum, palladium, and zinc oxide which have been proved as a potent remedy against a variety of infectious diseases along with other acute ailments [19,25]. The role of various phytochemicals such as alkaloids, flavonoids, phenols, sugars, proteins, and terpenoids has been confirmed in most of the previous reports emphasizing their involvement in the bioreduction, capping, and stabilization of metal ions $[26,27]$.

Despite the ease involved in the purification of NPs synthesized using only one single active substance in plant extract, it is important to further study the MNPs with a biomedical perspective for the treatment of particular diseases. At present, limited information is available in the scientific literature regarding the use of a single substance from plant extract for the synthesis of MNPs. Recent reports in literature on this issue show that the flavonoids which have a wide existence in the plant extracts have a major contribution toward the bioreduction, capping, and stabilization of metal ions into NPs formation [28-31].

\section{Plant extracts-mediated synthesis of NPs}

To prepare the plant extract, different parts of the plants are used as fresh or dry material such as the fruit, leaf, peel, petal, and shoot. The extraction procedure usually involves soaking the plant material in a green solvent with or without stirring followed by filtration and centrifugation. The filtered extract is rich in the reducing and capping agents required for the bioreduction of metallic ions. The advantage of using dried plant is that it has a long shelf life at room temperature, but it is important to store the fresh plant at $-20^{\circ} \mathrm{C}$ to avoid any deterioration. In addition, the use of dry plant material ensures the elimination of effects of seasonal variations leading to variations in plant constituents [32,33].

Various factors such as temperature, concentrations of the extract, and the metal ions and $\mathrm{pH}$ may affect the size and shape of the synthesized NPs [34]. The plant extract based synthesis procedures usually have 


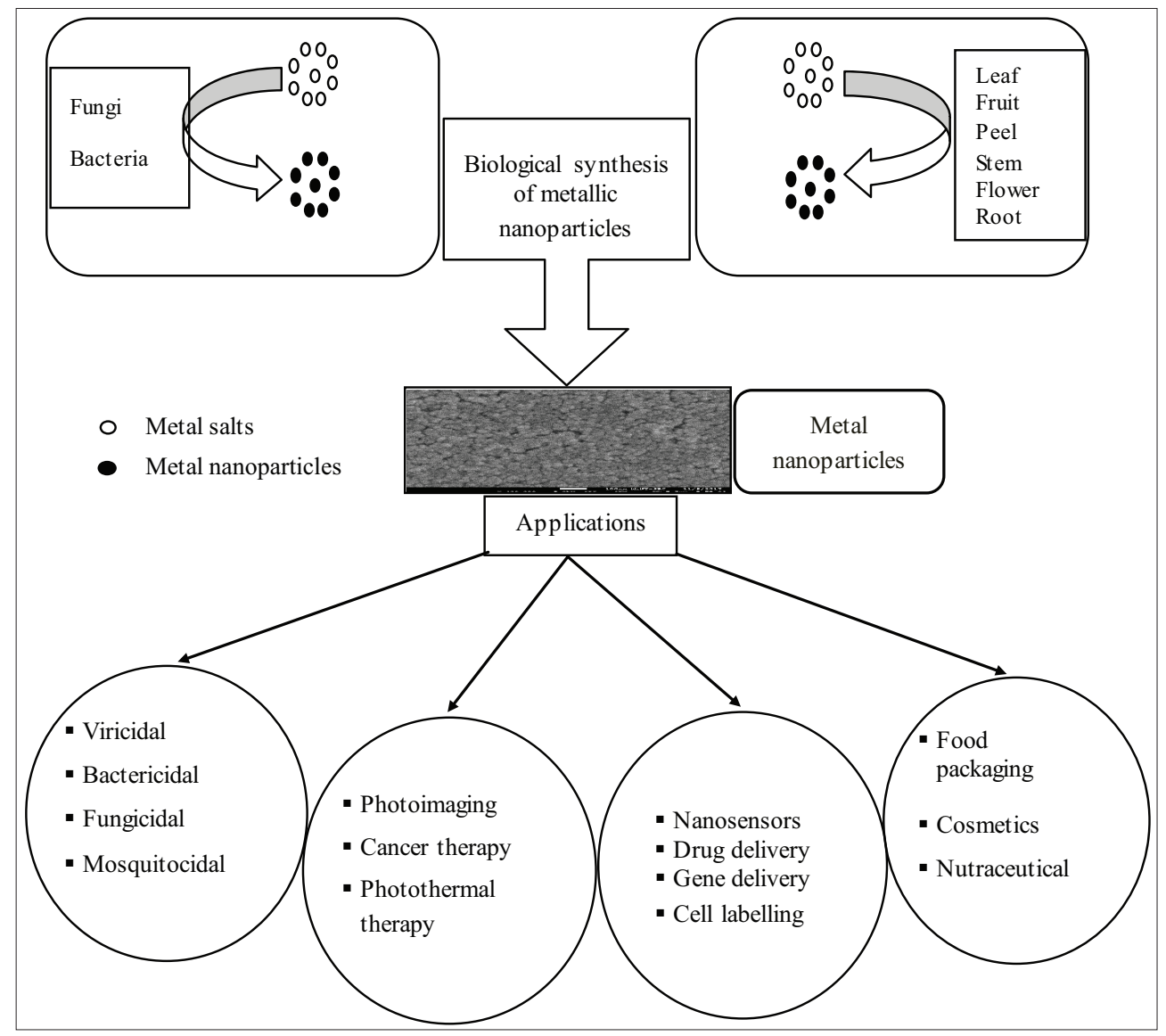

Fig. 2: Biological synthesis and applications of metal nanoparticles

a high rate of reaction, taking several minutes to several hours for completion, depending on the type and amount of the plant extract. Most plants, especially the perennial plants, are almost always naturally available. Usually, the plant extract-based synthesis of metallic NPs is carried out at room temperature, whereas heating of the reaction mixture or culture medium is required for the synthesis of metallic NPs using microorganisms. Due to the ease of handling and flexible reaction conditions, plant extract-mediated synthesis of MNPs is considered as more suitable for large-scale production as compared to microorganisms-based nanosynthesis $[5,35,36]$.

Polyphenols and flavonoids-based MNPs and their biomedical applications

The detail of polyphenols and flavonoids employed in the synthesis of MNPs along with their biomedical efficacy is summarized in Table 2. Recently, it was reported that the major contribution for the synthesis of silver NPs (AgNPs) was of the total flavonoids present in the Alternanthera tenella and Coriandrum sativum leaf extracts [7]; and shown to be efficacious as antiacne, antidandruff, and anti-breast cancer agent as they were found active against Propionibacterium acnes, Malassezia furfur, and human breast adenocarcinoma cells, respectively $[28,29]$.

The bioreduction of $\mathrm{Ag}^{+}$to AgNPs was carried out by the watersoluble flavonoids present in Myrmecodia pendan extract [37]. It was inferred that the flavonoids of Dalbergia spinosa leaf extract may be adsorbed onto the metal ions surface by interacting with carbonyl groups or electrons, thereby exhibiting increased anti-inflammatory, and antibacterial (against Bacillus subtilis, Pseudomonas aeruginosa, Staphylococcus aureus, and Escherichia coli) activities [38]. The flavonoids functionalized clove buds extract mediated AuNPs were reported to possess the anticancer activity against various cancer cells [39].

\section{Mechanisms of flavonoids-mediated NP synthesis}

There are some studies which proposed the plausible mechanism for polyphenols-mediated the synthesis of MNPs, as shown in Table 2. It was proposed that the hydroxyl groups present in the $\mathrm{B}$ and $\mathrm{C}$ rings of kaempferol participated in the AuNPs synthesis [76,112]. Moreover, the radical scavenging activity of NPs may be attributed to the A ring of kaempferol coating the surface of AuNPs. It was described that the formation of the enol form of luteolin, releasing reactive hydrogen, may be responsible for the reduction of $\mathrm{Ag}^{+}$to $\mathrm{Ag}^{0}$ [113].

It was proposed that the dihydromyricetin (DMY)-mediated synthesis of AuNPs occurred through the oxidation of hydroxyl to carbonyl groups. The study reported a shifting in the stretching vibration of the hydroxyl groups of DMY to higher wavenumber after bioreduction of Ag, which indicated the possible participation of hydroxyl in the reaction. In addition, there was a shift in the stretching vibration of carbonyl groups to lower wavenumber due to the oxidation of hydroxyl groups leading to the intramolecular hydrogen bonding [64].

Quercetin was found to chelate at three positions involving the carbonyl and hydroxyl groups at the C3 and C5 positions and the catechol group at the $\mathrm{C}^{\prime}$ and $\mathrm{C} 4^{\prime}$ positions. These groups were proposed to chelate different metal ions by the following steps: (1) Adsorption onto the metal surface, (2) budding of NP, (3) aggregation, and (4) bioreduction [59]. The possible mechanism for genistein AuNPs was proposed as follows: (1) Transfer of the electron from genistein into the $\mathrm{Au}$ center, (2) reduction of the $\mathrm{Au}^{3+}$ to $\mathrm{Au}^{0}$ by genistein, and (3) further acted as a stabilizing agent to form a layer of negative ions leading to the formation of the AuNPs [71].

The work of Kasthuri et al. [47] revealed that the reduction of $\mathrm{Au}^{3+} /$ $\mathrm{Ag}^{+}$ions occurred in a 2-step reaction involving the reduction by hydroxyl groups of the apiin followed by the oxidation of hydroxyl 
Table 2: Mechanism of synthesis and applications of MNPs

\begin{tabular}{|c|c|c|c|c|}
\hline Source & MNP (size) & Mechanism & Application & Reference \\
\hline $\begin{array}{l}\text { Abutilon indicum } \\
\text { (Polyphenols) }\end{array}$ & $\begin{array}{l}\text { AuNPs } \\
(1-20 \mathrm{~nm})\end{array}$ & $\begin{array}{l}\text { Hydroxyl and carbonyl groups of polyphenols mediated } \\
\text { bioreduction of Au metal ions }\end{array}$ & Anticancer & {$[40]$} \\
\hline $\begin{array}{l}\text { Acacia rigidula } \\
\text { (Phenolic compounds) }\end{array}$ & $\begin{array}{l}\text { AgNPs } \\
(8-66 \mathrm{~nm})\end{array}$ & $\begin{array}{l}\text { Hydroxyl groups of polyphenols mediated bioreduction } \\
\text { of Ag metal ions }\end{array}$ & Antibacterial & {$[41]$} \\
\hline $\begin{array}{l}\text { Acalypha indica } \\
\text { (Phenolic compounds) }\end{array}$ & $\begin{array}{l}\text { AgNPs } \\
(20-30 \mathrm{~nm})\end{array}$ & $\begin{array}{l}\text { Hydroxyl groups of phenolic compounds mediated } \\
\text { bioreduction of Ag metal ions }\end{array}$ & Antibacterial & {$[42]$} \\
\hline $\begin{array}{l}\text { Achyranthes aspera } \\
\text { (Phenolic compounds) }\end{array}$ & $\begin{array}{l}\text { AgNPs } \\
(7-14 \mathrm{~nm})\end{array}$ & $\begin{array}{l}\text { Hydroxyl groups of phenolic compounds mediated } \\
\text { bioreduction of Ag metal ions }\end{array}$ & Larvicidal against mosquito & {$[43]$} \\
\hline $\begin{array}{l}\text { Allium sativum } \\
\text { (Phenolic compounds) }\end{array}$ & $\begin{array}{l}\text { AgNPs } \\
(3-6 \mathrm{~nm})\end{array}$ & $\begin{array}{l}\text { Hydroxyl groups of polyphenols mediated bioreduction } \\
\text { of Ag metal ions }\end{array}$ & $\begin{array}{l}\text { Antibacterial } \\
\text { Antioxidant }\end{array}$ & {$[44]$} \\
\hline $\begin{array}{l}\text { Alpinia calcarata } \\
\text { (Phenolic compounds) }\end{array}$ & $\begin{array}{l}\text { AgNPs } \\
(27 \mathrm{~nm})\end{array}$ & $\begin{array}{l}\text { Hydroxyl groups of flavonoid/phenolic mediated } \\
\text { bioreduction of Ag metal ions }\end{array}$ & $\begin{array}{l}\text { Antibacterial } \\
\text { Antioxidant }\end{array}$ & {$[45]$} \\
\hline $\begin{array}{l}\text { Andean } \\
\text { blackberry } \\
\text { (Flavonoids) }\end{array}$ & $\begin{array}{l}\text { AgNPs } \\
(12-50 \mathrm{~nm})\end{array}$ & $\begin{array}{l}\text { Hydroxyl and carbonyl groups of flavonoid mediated } \\
\text { bioreduction of Ag metal ions }\end{array}$ & Antioxidant & {$[46]$} \\
\hline $\begin{array}{l}\text { Apiin (Apigenin } \\
\text { glycoside) }\end{array}$ & $\begin{array}{l}\text { AuNPs } \\
(21 \mathrm{~nm}) \\
\text { AgNPs } \\
(39 \mathrm{~nm})\end{array}$ & $\begin{array}{l}\text { Apiin hydroxyl groups-mediated reduction of metal ions } \\
\text { and subsequent formation of } \\
\text { carbonyl } \\
\text { groups that bind to metal ions leading to apiin coated } \\
\text { NPs }\end{array}$ & Anticancer & {$[47]$} \\
\hline $\begin{array}{l}\text { Azadirachta indica } \\
\text { (Phenolic compounds) }\end{array}$ & $\begin{array}{l}\text { AgNPs } \\
(34 \mathrm{~nm})\end{array}$ & $\begin{array}{l}\text { Hydroxyl groups of phenolic compounds mediated } \\
\text { reduction of Ag metal ions }\end{array}$ & - & [48] \\
\hline Baicalein & $\begin{array}{l}\text { AuNPs } \\
(26.5 \mathrm{~nm})\end{array}$ & $\begin{array}{l}\text { Hydroxyl groups of baicalein mediated reduction of } \mathrm{Au} \\
\text { metal ions - }\end{array}$ & $\begin{array}{l}\text { Antibiofilm activity against } \\
\text { P. aeruginosa }\end{array}$ & {$[49]$} \\
\hline Brassica oleracea & $\begin{array}{l}\text { AgNPs } \\
(24 \mathrm{~nm})\end{array}$ & $\begin{array}{l}\text { Hydroxyl groups of polyols-mediated reduction of Ag } \\
\text { metal ions }\end{array}$ & Cytotoxicity & {$[50]$} \\
\hline $\begin{array}{l}\text { Butea monosperma } \\
\text { (Polyphenols) }\end{array}$ & $\begin{array}{l}\text { AuNPs } \\
(10-30 \mathrm{~nm}) \\
\text { AgNPs } \\
(20-80 \mathrm{~nm})\end{array}$ & $\begin{array}{l}\text { Hydroxyl groups of polyphenols/protein-mediated } \\
\text { reduction of Au metal ions }\end{array}$ & Anticancer & {$[51]$} \\
\hline $\begin{array}{l}\text { Cassia fistula } \\
\text { (Flavonoids/ polyphenols) }\end{array}$ & $\begin{array}{l}\text { ZnNPs } \\
(5-15 \mathrm{~nm})\end{array}$ & 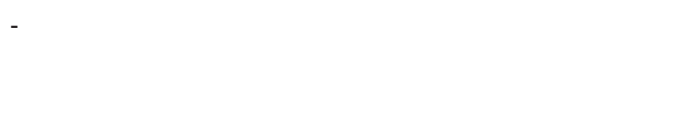 & $\begin{array}{l}\text { Dye reduction } \\
\text { Antioxidant } \\
\text { Antibacterial }\end{array}$ & {$[52]$} \\
\hline $\begin{array}{l}\text { Centella asiatica } \\
\text { (Flavonoids/polyphenols) }\end{array}$ & AuNPs & $\begin{array}{l}\text { Hydroxyl groups of flavonoids/polyphenols mediated } \\
\text { reduction of Au metal ions }\end{array}$ & - & {$[54]$} \\
\hline $\begin{array}{l}\text { Citrullus lanatus } \\
\text { (Phenolic compounds) }\end{array}$ & $\begin{array}{l}\text { AuNPs } \\
(20-140 \\
n m)\end{array}$ & $\begin{array}{l}\text { Hydroxyl groups of phenolic compounds mediated } \\
\text { reduction of } \mathrm{Au} \text { metal ions }\end{array}$ & $\begin{array}{l}\text { Antiproteasome } \\
\text { Antibacterial } \\
\text { Antioxidant }\end{array}$ & {$[55]$} \\
\hline $\begin{array}{l}\text { Citrus maxima (Flavonoids, } \\
\text { phenolic compounds) }\end{array}$ & $\begin{array}{l}\text { AuNPs } \\
(15-35 \mathrm{~nm})\end{array}$ & $\begin{array}{l}\text { Hydroxyl and amide groups mediated reduction of } \mathrm{Au} \\
\text { metal ions }\end{array}$ & Catalytic dye reduction & {$[56]$} \\
\hline $\begin{array}{l}\text { Citrus sinensis } \\
\text { (Phenolic compounds) }\end{array}$ & $\begin{array}{l}\text { AgNPs } \\
(10-35 \mathrm{~nm})\end{array}$ & $\begin{array}{l}\text { Hydroxyl groups of phenolic compounds mediated } \\
\text { reduction of Ag metal ions }\end{array}$ & Antibacterial & {$[57]$} \\
\hline $\begin{array}{l}\text { Clove buds } \\
\text { (Flavonoids) }\end{array}$ & $\begin{array}{l}\text { AuNPs and } \\
\text { AgNPs }\end{array}$ & - & $\begin{array}{l}\text { Anticancer against cancer } \\
\text { of the human cervix, human } \\
\text { chronic myelogenous } \\
\text { leukemia, } \\
\text { human colorectal } \\
\text { adenocarcinoma, and } \\
\text { human renal carcinoma }\end{array}$ & {$[39]$} \\
\hline $\begin{array}{l}\text { Coleus aromaticus } \\
\text { (Phenolic compounds) }\end{array}$ & $\begin{array}{l}\text { AgNPs } \\
(44 \mathrm{~nm})\end{array}$ & $\begin{array}{l}\text { Hydroxyl groups of phenolic compounds mediated } \\
\text { reduction of Ag metal ions }\end{array}$ & Antibacterial & {$[58]$} \\
\hline $\begin{array}{l}\text { Coriandrum } \\
\text { sativum (Flavonoids) }\end{array}$ & $\begin{array}{l}\text { AgNPs } \\
(37 \mathrm{~nm})\end{array}$ & Hydroxyl groups mediated reduction of Ag metal ions- & $\begin{array}{l}\text { Antiacne } \\
\text { (Propionibacterium acnes), } \\
\text { antidandruff (Malassezia } \\
\text { furfur) and } \\
\text { anticancer against human } \\
\text { breast cancer }\end{array}$ & {$[28]$} \\
\hline $\begin{array}{l}\text { Coriandrum } \\
\text { sativum (Luteolin) }\end{array}$ & $\begin{array}{l}\text { AgNPs } \\
(13 \mathrm{~nm})\end{array}$ & $\begin{array}{l}\text { Conversion of } \mathrm{Ag}^{+} \text {to } \mathrm{Ag}^{0} \text { by the freely released reactive } \\
\text { hydrogen from the enol form of luteolin. }\end{array}$ & $\begin{array}{l}\text { Antibacterial against } \\
\text { B. subtilis }\end{array}$ & [59] \\
\hline $\begin{array}{l}\text { Costus afer } \\
\text { (Flavonoids, phenolic } \\
\text { compounds) }\end{array}$ & $\begin{array}{l}\text { AgNPs } \\
(5-38 \mathrm{~nm})\end{array}$ & $\begin{array}{l}\text { Hydroxyl groups of flavonoids and phenolic compounds } \\
\text { mediated reduction of Ag metal ions }\end{array}$ & $\begin{array}{l}\text { Antibacterial } \\
\text { Antioxidant }\end{array}$ & {$[60]$} \\
\hline $\begin{array}{l}\text { Curcuma longa } \\
\text { (Phenolic compounds) }\end{array}$ & $\begin{array}{l}\text { AgNPs } \\
(58 \mathrm{~nm})\end{array}$ & $\begin{array}{l}\text { Hydroxyl groups of phenolic compounds mediated } \\
\text { reduction of Ag metal ions }\end{array}$ & Anticancer & {$[61]$} \\
\hline
\end{tabular}


Table 2: (Continued)

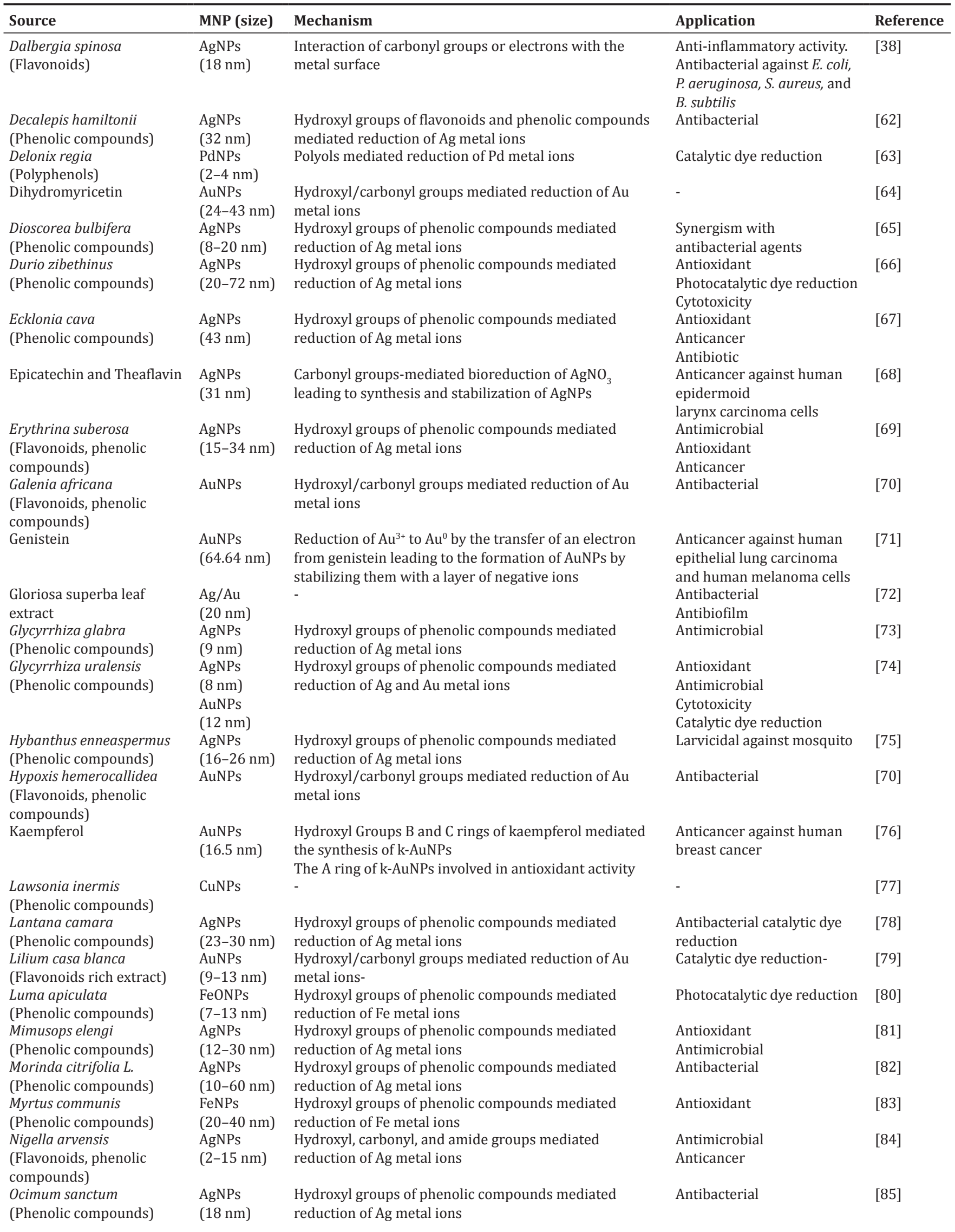


Table 2: (Continued)

\begin{tabular}{|c|c|c|c|c|}
\hline Source & MNP (size) & Mechanism & Application & Reference \\
\hline $\begin{array}{l}\text { Potentilla fulgens } \\
\text { (Flavonoids) }\end{array}$ & $\begin{array}{l}\text { AgNPs } \\
(10-15 \mathrm{~nm})\end{array}$ & $\begin{array}{l}\text { Hydroxyl, carbonyl, and amide groups mediated } \\
\text { reduction of Ag metal ions }\end{array}$ & $\begin{array}{l}\text { Anticancer against human } \\
\text { breast cancer and human } \\
\text { glioblastoma cancer cells } \\
\text { Antibacterial against } E \text {. coli } \\
\text { and } B \text {. subtilis }\end{array}$ & [86] \\
\hline Proanthocyanidin & $\begin{array}{l}\text { AuNPs } \\
(17-29 \mathrm{~nm})\end{array}$ & $\begin{array}{l}\text { Hydroxyl/carbonyl groups mediated reduction of Ag } \\
\text { metal ions }\end{array}$ & $\begin{array}{l}\text { The efficient } \\
\text { cardioprotective potential } \\
\text { with good biocompatibility }\end{array}$ & [87] \\
\hline $\begin{array}{l}\text { Pueraria tuberosa } \\
\text { (Flavonoids/phenolic } \\
\text { compounds) }\end{array}$ & $\begin{array}{l}\text { AgNPs } \\
(162 \mathrm{~nm})\end{array}$ & $\begin{array}{l}\text { Hydroxyl groups of flavonoids/phenolic compounds } \\
\text { mediated reduction of Ag metal ions }\end{array}$ & $\begin{array}{l}\text { Antioxidant } \\
\text { Anticancer }\end{array}$ & {$[88]$} \\
\hline $\begin{array}{l}\text { Pulicaria glutinosa } \\
\text { (Phenolic compounds) }\end{array}$ & $\begin{array}{l}\text { AgNPs } \\
(40-60 \mathrm{~nm})\end{array}$ & $\begin{array}{l}\text { Hydroxyl groups of phenolic compounds mediated } \\
\text { reduction of Ag metal ions }\end{array}$ & - & [89] \\
\hline $\begin{array}{l}\text { Punica granatum } \\
\text { (Flavonoids, phenolic } \\
\text { compounds) }\end{array}$ & $\begin{array}{l}\text { AgNPs } \\
(40-70 \mathrm{~nm})\end{array}$ & $\begin{array}{l}\text { Hydroxyl groups of flavonoids/polyphenols mediated } \\
\text { reduction of Ag metal ions }\end{array}$ & Anticancer & {$[90]$} \\
\hline Quercetin & $\begin{array}{l}\text { Ag-SeNPs } \\
(30-35 \mathrm{~nm})\end{array}$ & $\begin{array}{l}\text { Hydroxyl/carbonyl groups mediated reduction of } \mathrm{Ag} / \mathrm{Se} \\
\text { metal ions }\end{array}$ & $\begin{array}{l}\text { Antioxidant, antimicrobial, } \\
\text { and anticancer activities }\end{array}$ & [91] \\
\hline Quercetin & $\begin{array}{l}\text { Metal } \\
\text { NPs }\end{array}$ & $\begin{array}{l}\text { Metal ion chelation by three positions of quercetin } \\
\text { involving the carbonyl and hydroxyl groups at the C } 3 \\
\text { and C5 positions and the catechol group at the C } 30 \text { and } \\
\text { C40 site }\end{array}$ & - & [59] \\
\hline $\begin{array}{l}\text { Quercus brantii } \\
\text { (Phenolic compounds) }\end{array}$ & $\begin{array}{l}\text { AgNPs } \\
(6 \mathrm{~nm})\end{array}$ & $\begin{array}{l}\text { Hydroxyl groups of flavonoids/polyphenols mediated } \\
\text { reduction of Ag metal ions }\end{array}$ & - & {$[92]$} \\
\hline $\begin{array}{l}\text { Ranunculus muricatus } \\
\text { (Flavonoids) }\end{array}$ & $\begin{array}{l}\mathrm{Au} / \mathrm{TiO}_{2} \\
(50-90 \mathrm{~nm})\end{array}$ & $\begin{array}{l}\text { Hydroxyl groups interact with metal ions through a } \\
\text { covalent bond }\end{array}$ & $\begin{array}{l}\text { Antibacterial against } \\
\text { S. aureus and E. coli }\end{array}$ & [93] \\
\hline $\begin{array}{l}\text { Rosmarinus officinalis } \\
\text { (Phenolic compounds) }\end{array}$ & $\begin{array}{l}\text { AgNPs } \\
(10-30 \mathrm{~nm})\end{array}$ & $\begin{array}{l}\text { Hydroxyl groups of phenolic compounds mediated } \\
\text { reduction of Ag metal ions }\end{array}$ & Antimicrobial & {$[94]$} \\
\hline $\begin{array}{l}\text { Salacia chinensis } \\
\text { (Phenolic compounds) }\end{array}$ & $\begin{array}{l}\text { AgNPs } \\
(40-80 \mathrm{~nm})\end{array}$ & $\begin{array}{l}\text { Hydroxyl groups of phenolic compounds mediated } \\
\text { reduction of Ag metal ions }\end{array}$ & Anticancer & [95] \\
\hline $\begin{array}{l}\text { Satureja intermedia } \\
\text { (Phenolic compounds) }\end{array}$ & $\begin{array}{l}\text { AgNPs } \\
(28 \mathrm{~nm})\end{array}$ & $\begin{array}{l}\text { Hydroxyl groups of phenolic compounds mediated } \\
\text { reduction of Ag metal ions }\end{array}$ & Antioxidant & [96] \\
\hline $\begin{array}{l}\text { Sesbania grandiflora } \\
\text { (Flavonoids, polyphenols) }\end{array}$ & $\begin{array}{l}\text { AuNPs } \\
(7-34 \mathrm{~nm})\end{array}$ & $\begin{array}{l}\text { Hydroxyl/carbonyl groups mediated reduction of } \mathrm{Au} \\
\text { metal ions }\end{array}$ & Catalytic dye reduction & [97] \\
\hline $\begin{array}{l}\text { Sterculia acuminata } \\
\text { (Polyphenols) }\end{array}$ & $\begin{array}{l}\text { AuNPs } \\
(9-38 \mathrm{~nm})\end{array}$ & $\begin{array}{l}\text { Hydroxyl groups of phenolic compounds mediated } \\
\text { reduction of Au metal ions }\end{array}$ & Catalytic dye reduction & [99] \\
\hline $\begin{array}{l}\text { Suaeda monoica } \\
\text { (Phenolic compounds) }\end{array}$ & $\begin{array}{l}\text { AuNPs } \\
(3-25 \mathrm{~nm})\end{array}$ & $\begin{array}{l}\text { Hydroxyl groups of phenolic compounds mediated } \\
\text { reduction of Au metal ions }\end{array}$ & Antioxidant & {$[100]$} \\
\hline $\begin{array}{l}\text { Sunflower oil } \\
\text { (Phenolic compounds) }\end{array}$ & $\begin{array}{l}\text { AgNPs } \\
(50 \mathrm{~nm})\end{array}$ & $\begin{array}{l}\text { Hydroxyl groups of phenolic compounds mediated } \\
\text { reduction of Ag metal ions }\end{array}$ & Anticancer & {$[101]$} \\
\hline $\begin{array}{l}\text { Syzygium cumini } \\
\text { (Phenolic compounds) }\end{array}$ & $\begin{array}{l}\text { AgNPs } \\
(20-60 \mathrm{~nm})\end{array}$ & $\begin{array}{l}\text { Hydroxyl groups of phenolic compounds mediated } \\
\text { reduction of Ag metal ions }\end{array}$ & Antibacterial & [102] \\
\hline $\begin{array}{l}\text { Syzygium cumini } \\
\text { (Phenolic compounds) }\end{array}$ & $\begin{array}{l}\text { AgNPs } \\
(30-92 \mathrm{~nm})\end{array}$ & $\begin{array}{l}\text { Hydroxyl groups of phenolic compounds mediated } \\
\text { reduction of Ag metal ions }\end{array}$ & - & [103] \\
\hline Syzygium samarangense & AgNPs & $\begin{array}{l}\text { Hydroxyl groups of phenolic compounds mediated } \\
\text { reduction of Ag metal ions }\end{array}$ & Anticancer & {$[104]$} \\
\hline $\begin{array}{l}\text { Tamarindus indica } \\
\text { (Phenolic compounds) }\end{array}$ & $\begin{array}{l}\text { AuNPs } \\
(52 \mathrm{~nm})\end{array}$ & $\begin{array}{l}\text { Hydroxyl/carboxylic groups mediated reduction of } \mathrm{Au} \\
\text { metal ions }\end{array}$ & - & {$[105]$} \\
\hline $\begin{array}{l}\text { Tea extract } \\
\text { (Phenolic compounds) }\end{array}$ & $\begin{array}{l}\text { AuNPs } \\
(8-24 \mathrm{~nm})\end{array}$ & $\begin{array}{l}\text { Hydroxyl groups of phenolic compounds mediated } \\
\text { reduction of Ag metal ions }\end{array}$ & Catalytic dye reduction & {$[106]$} \\
\hline $\begin{array}{l}\text { Tephrosia } \\
\text { tinctoria (Flavonoids) }\end{array}$ & $\begin{array}{l}\text { AgNPs } \\
(<100 \mathrm{~nm})\end{array}$ & $\begin{array}{l}\text { Hydroxyl/carboxylic groups mediated reduction of } \mathrm{Au} \\
\text { metal ions }\end{array}$ & Antidiabetic & {$[107]$} \\
\hline $\begin{array}{l}\text { Terminalia arjuna } \\
\text { (Polyphenols) }\end{array}$ & $\begin{array}{l}\text { AuNPs } \\
(20-50 \mathrm{~nm})\end{array}$ & $\begin{array}{l}\text { Hydroxyl/carboxylic groups mediated reduction of } \mathrm{Au} \\
\text { metal ions }\end{array}$ & $\begin{array}{l}\text { Enhancement of seed } \\
\text { germination activity in } \\
\text { Gloriosa superba }\end{array}$ & {$[108]$} \\
\hline $\begin{array}{l}\text { Terminalia catappa } \\
\text { (Phenols) }\end{array}$ & $\begin{array}{l}\text { AuNPs } \\
(10-35 \mathrm{~nm})\end{array}$ & $\begin{array}{l}\text { Hydroxyl/carbonyl groups mediated reduction of } \mathrm{Au} \\
\text { metal ions }\end{array}$ & - & [109] \\
\hline $\begin{array}{l}\text { Walnut green husk } \\
\text { (Polyphenols) }\end{array}$ & $\begin{array}{l}\text { AgNPs } \\
(31.4 \mathrm{~nm})\end{array}$ & $\begin{array}{l}\text { Hydroxyl groups of polyphenols mediated reduction of } \\
\text { Ag metal ions }\end{array}$ & $\begin{array}{l}\text { Antioxidant } \\
\text { Antimicrobial } \\
\text { Anticancer }\end{array}$ & [110] \\
\hline $\begin{array}{l}\text { Zingiber officinale } \\
\text { (Phenolic compounds) }\end{array}$ & $\begin{array}{l}\text { AgNPs } \\
(10-20 \mathrm{~nm})\end{array}$ & $\begin{array}{l}\text { Hydroxyl groups of polyphenols mediated reduction of } \\
\text { Ag metal ions }\end{array}$ & Antibacterial & {$[111]$} \\
\hline
\end{tabular}

B. subtilis: Bacillus subtilis, P. aeruginosa: Pseudomonas aeruginosa, S. aureus: Staphylococcus aureus, E. coli: Escherichia coli, MNP: Metal nanoparticle, AgNPs: Silver nanoparticles 


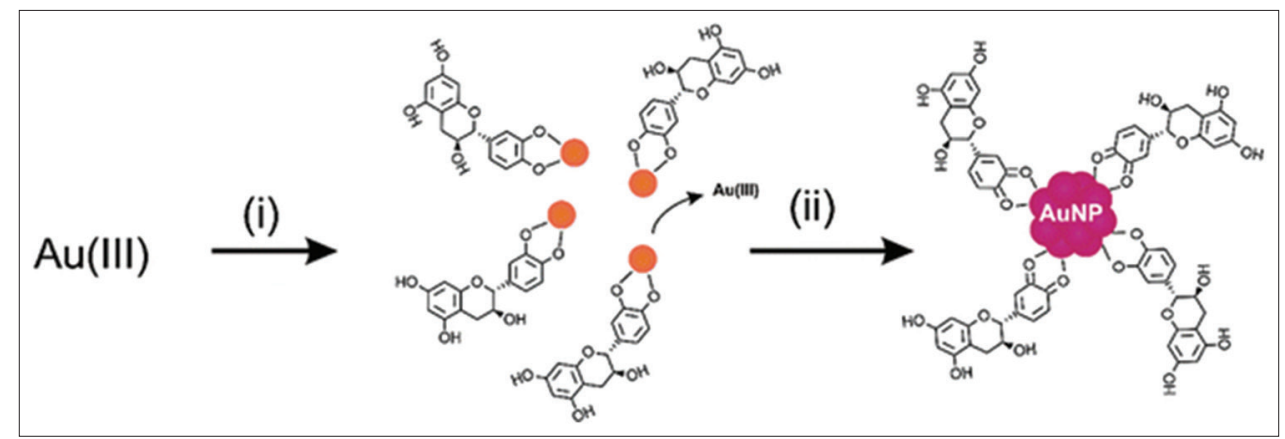

Fig. 3: Mechanism of polyphenols-mediated GNP synthesis: (1) Polyphenols and (2) synthesis and stabilization of gold nanoparticles [114]

groups to the carbonyl groups. Finally, the binding of carbonyl groups of apiin to the metal ion, thereby coating the NPs surfaces to prevent agglomeration. Most of these cited references from available literature give a clear indication that both the hydroxyl and the carbonyl groups of polyphenols collectively play a key role in the formation of MNPs.

Fig. 3 depicts the mechanism of polyphenols-based GNP synthesis. The adjacent hydroxyl groups of polyphenolic compounds from a 5-member chelate ring structure followed by oxidation of the chelated dihydroxy groups to quinones. Due to the high oxidation-reduction potential of $\mathrm{Au}^{3+}$, the quinones subsequently reduce the gold metal ions from $\mathrm{Au}^{3+}$ to $\mathrm{Au}^{0}$. The synthesis of gold NPs occurs after a collision between the adjacent $\mathrm{Au}^{0}$ atoms, and the NPs thus formed are stabilized by polyphenolic compounds including the quinones [114].

\section{CONCLUSION}

The emerging threats related to the toxic and hazardous nature of the conventional methods of NP synthesis have led to the plant extractsmediated synthesis of MNPs. The green nanosynthesis approach thus adopted is cost and time effective, and environment-friendly with the potential to easily scale up the product. Such a non-toxic approach is especially desirable to synthesize the NPs that must not be toxic if they are destined for the therapeutic applications. The NPs of controlled size and shape can be synthesized using various plant extracts of which the polyphenols, including flavonoids, are considered as the most active bioreductants of metal ions. The MNPs synthesized using natural polyphenols and flavonoids have shown a number of biomedical applications, including their therapeutic activity against various ailments.

\section{ACKNOWLEDGMENT}

This study was financially supported by the Research Management Centre (RMC), Universiti Tun Hussein Onn Malaysia through TIER-1 Vot No. H256 and Geran Penyelidikan Pascasiswazah (GPPS) Vot. No. U490.

\section{AUTHORS' CONTRIBUTIONS}

All the authors of this review paper have contributed equally in retrieving, collecting, and compiling the data as well as writing and proofreading of the manuscript.

\section{CONFLICTS OF INTEREST}

The authors declare that there are no conflicts of interest regarding the publication of this review paper.

\section{REFERENCES}

1. Zhang XF, Liu ZG, Shen W, Gurunathan S. Silver nanoparticles: Synthesis, characterization, properties, applications, and therapeutic approaches. Int J Mol Sci 2016;17:E1534.

2. Bundschuh M, Filser J, Lüderwald S, McKee MS, Metreveli G, Schaumann GE, et al. Nanoparticles in the environment: Where do we come from, where do we go to? Environ Sci Eur 2018;30:6.

3. Hussain I, Singh NB, Singh A, Singh H, Singh SC. Green synthesis of nanoparticles and its potential application. Biotechnol Lett 2016;38:545-60.

4. Saratale RG, Saratale GD, Shin HS, Jacob JM, Pugazhendhi A, Bhaisare $\mathrm{M}$, et al. New insights on the green synthesis of metallic nanoparticles using plant and waste biomaterials: Current knowledge, their agricultural and environmental applications. Environ Sci Pollut Res Int 2018;25:10164-83.

5. Iravani S. Methods for preparation of metal nanoparticles. In: Thota S, Debbie CC, editors. Metal Nanoparticles: Synthesis and Applications in Pharmaceutical Sciences. USA: Wiley-VCH Verlag; 2018. p. 15-32.

6. Shah M, Fawcett D, Sharma S, Tripathy SK, Poinern GE. Green synthesis of metallic nanoparticles via biological entities. Materials (Basel) 2015;8:7278-308.

7. Sathishkumar P, Gu FL, Zhan Q, Palvannan T, Yusoff AR. Flavonoids mediated 'green' nanomaterials: A novel nanomedicine system to treat various diseases current trends and future perspective. Mater Lett 2018;210:26-30.

8. Santhoshkumar J, Rajeshkumar S, Kumar SV. Phyto-assisted synthesis, characterization and applications of gold nanoparticles-a review. Biochem Biophys Rep 2017;11:46-57.

9. Singh P, Kim YJ, Zhang D, Yang DC. Biological synthesis of nanoparticles from plants and microorganisms. Trends Biotechnol 2016;34:588-99.

10. Ovais M, Khalil AT, Islam NU, Ahmad I, Ayaz M, Saravanan M, et al. Role of plant phytochemicals and microbial enzymes in biosynthesis of metallic nanoparticles. Appl Microbiol Biotechnol 2018;102:6799814.

11. Ahmed S, Annu, Ikram S, Yudha SS. Biosynthesis of gold nanoparticles: A green approach. J Photochem Photobiol B 2016;161:141-53.

12. Fakruddin M, Hossain Z, Afroz H. Prospects and applications of nanobiotechnology: A medical perspective. J Nanobiotechnology 2012;10:31.

13. de Marco BA, Rechelo BS, Tótoli EG, Kogawa AC, Salgado HR. Evolution of green chemistry and its multidimensional impacts: A review. Saudi Pharm J 2019;27:1-8.

14. Gilbertson LM, Zimmerman JB, Plata DL, Hutchison JE, Anastas PT. Designing nanomaterials to maximize performance and minimize undesirable implications guided by the principles of green chemistry. Chem Soc Rev 2015;44:5758-77.

15. Kratošová G, Holišová V, Konvičková Z, Ingle AP, Gaikwad S, Škrlová K, et al. From biotechnology principles to functional and lowcost metallic bionanocatalysts. Biotechnol Adv 2019;37:154-76.

16. Kalpana VN, Rajeswari VD. A review on green synthesis, biomedical applications, and toxicity studies of znO NPs. Bioinorg Chem Appl 2018;2018:3569758.

17. Das RK, Pachapur VL, Lonappan L, Naghdi M, Pulicharla R, Maiti $\mathrm{S}$, et al. Biological synthesis of metallic nanoparticles: Plants, animals and microbial aspects. Nanotechnol Environ Eng 2017;2:18.

18. Khan I, Saeed K, Khan I. Nanoparticles: Properties, applications and toxicities. Arabian J Chem 2017. Doi: 10.1016/j.arabjc.2017.05.011.

19. Kuppusamy P, Yusoff MM, Maniam GP, Govindan N. Biosynthesis of metallic nanoparticles using plant derivatives and their new avenues in pharmacological applications-an updated report. Saudi Pharm J 2016;34:473-84.

20. Thakkar KN, Mhatre SS, Parikh RY. Biological synthesis of metallic nanoparticles. Nanomedicine 2010;6:257-62.

21. Reddy NJ, Nagoor Vali D, Rani M, Rani SS. Evaluation of antioxidant, antibacterial and cytotoxic effects of green synthesized silver nanoparticles by Piper longum fruit. Mater Sci Eng C Mater Biol Appl 2014;34:115-22. 
22. Rehana D, Mahendiran D, Kumar RS, Rahiman AK. In vitro antioxidant and antidiabetic activities of zinc oxide nanoparticles synthesized using different plant extracts. Bioprocess Biosyst Eng 2017;40:943-57.

23. Fahimirad S, Ajalloueian F, Ghorbanpour M. Synthesis and therapeutic potential of silver nanomaterials derived from plant extracts. Ecotoxicol Environ Saf 2019;168:260-78.

24. Rao PV, Nallappan D, Madhavi K, Rahman S, Jun Wei L, Gan SH, et al. Phytochemicals and biogenic metallic nanoparticles as anticancer agents. Oxid Med Cell Longev 2016;2016:3685671.

25. Ovais M, Raza A, Naz S, Islam NU, Khalil AT, Ali S, et al. Current state and prospects of the phytosynthesized colloidal gold nanoparticles and their applications in cancer theranostics. Appl Microbiol Biotechnol 2017;101:3551-65.

26. Jayaprakash N, Vijaya JJ, Kaviyarasu K, Kombaiah K, Kennedy LJ, Ramalingam RJ, et al. Green synthesis of ag nanoparticles using tamarind fruit extract for the antibacterial studies. J Photochem Photobiol B 2017; 169:178-85.

27. Khodadadi B, Bordbar M, Nasrollahzadeh M. Green synthesis of pd nanoparticles at apricot kernel shell substrate using Salvia hydrangea extract: Catalytic activity for reduction of organic dyes. J Colloid Interface Sci 2017;490:1-10

28. Sathishkumar P, Preethi J, Vijayan R, Mohd Yusoff AR, Ameen F, Suresh S, et al. Anti-acne, anti-dandruff and anti-breast cancer efficacy of green synthesised silver nanoparticles using Coriandrum sativum leaf extract. J Photochem Photobiol B 2016;163:69-76.

29. Sathishkumar P, Vennila K, Jayakumar R, Yusoff AR, Hadibarata T, Palvannan T, et al. Phyto-synthesis of silver nanoparticles using Alternanthera tenella leaf extract: An effective inhibitor for the migration of human breast adenocarcinoma (MCF-7) cells. Bioprocess Biosyst Eng 2016;39:651-9.

30. Marslin G, Siram K, Maqbool Q, Selvakesavan RK, Kruszka D, Kachlicki P, et al. Secondary metabolites in the green synthesis of metallic nanoparticles. Materials (Basel) 2018;11:E940.

31. Jain S, Mehata MS. Medicinal plant leaf extract and pure flavonoid mediated green synthesis of silver nanoparticles and their enhanced antibacterial property. Sci Rep 2017;7:15867.

32. Ingle KP, Deshmukh AG, Padole DA, Dudhare MS, Moharil MP, Vaibhav KC. Phytochemicals: Extraction methods, identification and detection of bioactive compounds from plant extracts. J Pharmacogn Phytochem 2017;6:32-6.

33. Altemimi A, Lakhssassi N, Baharlouei A, Watson DG, Lightfoot DA. Phytochemicals: Extraction, isolation, and identification of bioactive compounds from plant extracts. Plants (Basel) 2017;6:42.

34. Vijayaraghavanab K, Ashokkumar T. Plant-mediated biosynthesis of metallic nanoparticles: A review of literature, factors affecting synthesis, characterization techniques and applications. J Environ Chem Eng 2017;5:4866-83.

35. Kumar V, Yadav SK. Plant-mediated synthesis of silver and gold nanoparticles and their applications. J Chem Biotechnol 2009;84:151-7.

36. Ahmed S, Ahmad M, Swami BL, Ikram S. A review on plants extract mediated synthesis of silver nanoparticles for antimicrobial applications: A green expertise. J Adv Res 2016;7:17-28.

37. Zuas O, Hamim N, Sampora Y. Bio-synthesis of silver nanoparticles using water extract of Myrmecodia pendan (sarang semut plant). Mater Lett 2014;123:156-9.

38. Muniyappan N, Nagarajan NS. Green synthesis of silver nanoparticles with Dalbergia spinosa leaves and their applications in biological and catalytic activities. Process Biochem 2014;49:1054-61.

39. Raghunandan D, Ravishankar B, Sharanbasava G, Mahesh DB, Harsoor V, Yalagatti MS, et al. Anti-cancer studies of noble metal nanoparticles synthesized using different plant extracts. Cancer Nanotechnol 2011;2:57-65.

40. Mata R, Nakkala JR, Sadras SR. Polyphenol stabilized colloidal gold nanoparticles from Abutilon indicum leaf extract induce apoptosis in HT-29 colon cancer cells. Colloids Surf B Biointerfaces 2016;143:499-510.

41. Escárcega-González CE, Garza-Cervantes JA, Vázquez-Rodríguez A, Montelongo-Peralta LZ, Treviño-González MT, Díaz Barriga Castro $\mathrm{E}$, et al. In vivo antimicrobial activity of silver nanoparticles produced via a green chemistry synthesis using Acacia rigidula as a reducing and capping agent. Int J Nanomedicine 2018;13:2349-63.

42. Krishnaraj C, Jagan EG, Rajasekar S, Selvakumar P, Kalaichelvan PT, Mohan N, et al. Synthesis of silver nanoparticles using Acalypha indica leaf extracts and its antibacterial activity against water borne pathogens. Colloids Surf B Biointerfaces 2010;76:50-6.

43. Elumalai D, Kaleena PK, Ashok K, Suresh A, Hemavathi M. Green synthesis of silver nanoparticle using Achyranthes aspera and its larvicidal activity against three major mosquito vectors. Eng Agric Environ Food 2016;9:1-8

44. Otunola GA, Afolayan AJ, Ajayi EO, Odeyemi SW. Characterization, antibacterial and antioxidant properties of silver nanoparticles synthesized from aqueous extracts of Allium sativum, Zingiber officinale, and Capsicum frutescens. Pharmacogn Mag 2017;13:S201-8.

45. Khandel P, Shahi SK, Soni DK, Yadaw RK, Kanwar L. Alpinia calcarata: Potential source for the fabrication of bioactive silver nanoparticles. Nano Converg 2018;5:37.

46. Kumar B, Smita K, Cumbal L, Debut A. Green synthesis of silver nanoparticles using andean blackberry fruit extract. Saudi J Biol Sci 2017;24:45-50

47. Kasthuri J, Veerapandian S, Rajendiran N. Biological synthesis of silver and gold nanoparticles using apiin as reducing agent. Colloids Surf B Biointerfaces 2009;68:55-60.

48. Ahmed S, Saifullah MA, Ahmad M, Swamy BL, Ikram S. Green synthesis of silver nanoparticles using Azadirachta indica aqueous leaf extract. J Radiat Res Appl Sci 2016;9:1-7.

49. Rajkumari J, Busi S, Vasu AC, Reddy P. Facile green synthesis of baicalein fabricated gold nanoparticles and their antibiofilm activity against Pseudomonas aeruginosa PAO1. Microb Pathog 2017;107:261-9.

50. Lathamuthiah B, Inbakandan, Devi R. In vivo toxicity studies of biosynthesized silver nanoparticles using Brassica oleracea in zebra fish model. Int J Pharm Pharm Sci 2015;7:425-30.

51. Patra S, Mukherjee S, Barui AK, Ganguly A, Sreedhar B, Patra CR, et al. Green synthesis, characterization of gold and silver nanoparticles and their potential application for cancer therapeutics. Mater Sci Eng C Mater Biol Appl 2015;53:298-309.

52. Tewari D, Mukhopadhyay M, Nekkanti MS, Vallabhaneni S, Sahu G, Jetti SK, et al. Cytoprotective effect of Centella asiatica is mediated through the modulation of mitochondrial voltage-dependent anion channel (VDAC) and scavenging of free radicals. J Funct Foods 2016;21:301-11.

53. Halevas E, Nday CM, Salifoglou A. Hybrid catechin silica nanoparticle influence on $\mathrm{cu}(\mathrm{II})$ toxicity and morphological lesions in primary neuronal cells. J Inorg Biochem 2016;163:240-9.

54. Latif MS, Kormin F, Mustafa MK, Khan M, Mohamad II, Abbas $\mathrm{S}$, et al. Effect of temperature on the synthesis of Centella asiatica flavonoids extract-mediated gold nanoparticles: UV-visible spectra analyses. AIP Conf Proc 2018;2016:20071.

55. Patra JK, Baek KH. Novel green synthesis of gold nanoparticles using Citrullus lanatus rind and investigation of proteasome inhibitory activity, antibacterial, and antioxidant potential. Int J Nanomedicine 2015;10:7253-64.

56. Yu J, Xu D, Guan HN, Wang C, Huang LK, Chi DF. Facile one-step green synthesis of gold nanoparticles using Citrus maxima aqueous extracts and its catalytic activity. Mater Lett 2016;166:110-2.

57. Kaviya S, Santhanalakshmi J, Viswanathan B, Muthumary J, Srinivasan K. Biosynthesis of silver nanoparticles using Citrus sinensis peel extract and its antibacterial activity. Spectrochim Acta A Mol Biomol Spectrosc 2011;79:594-8.

58. Vanaja M, Annadurayi G. Coleus aromaticus leaf extract mediated synthesis of silver nanoparticles and its bactericidal activity. Appl Nanosci 2013;3:217.

59. Makarov VV, Love AJ, Sinitsyna OV, Makarova SS, Yaminsky IV, Taliansky ME, et al. "Green" nanotechnologies: Synthesis of metal nanoparticles using plants. Acta Naturae 2014;6:35-44.

60. Elemike EE, Fayemi OE, Ekennia AC, Onwudiwe DC, Ebenso EE. Silver nanoparticles mediated by Costus afer leaf extract: Synthesis, antibacterial, antioxidant and electrochemical properties. Molecules 2017;22:701.

61. Sheikh E, Bhatt ML, Tripathi M. Bio-based synthesised and characterized monodispersed Curcuma longa silver nanoparticles induces targeted anticancer activity in breast cancer cells. Pharmacogn Mag 2018; 14:340-41

62. Rashmi V, Sanjay KR. Green synthesis, characterisation and bioactivity of plant-mediated silver nanoparticles using Decalepis hamiltonii root extract. IET Nanobiotechnol 2017;11:247-54.

63. Dauthal P, Mukhopadhyay M. Biosynthesis of palladium nanoparticles using Delonix regia leaf extract and its catalytic activity for nitroaromatics hydrogenation. Ind Eng Chem Res 2013;52:18131-9.

64. Guo Q, Guo Q, Yuan J, Zeng J. Biosynthesis of gold nanoparticles using a kind of flavonol: Dihydromyricetin. Colloids Surf A Physicochem Eng Aspects 2014;441:127-32.

65. Ghosh S, Patil S, Ahire M, Kitture R, Kale S, Pardesi K, et al. 
Synthesis of silver nanoparticles using Dioscorea bulbifera tuber extract and evaluation of its synergistic potential in combination with antimicrobial agents. Int J Nanomedicine 2012;7:483-96.

66. Sumitha S, Vasanthi S, Shalini S, Chinni SV, Gopinath SC, Anbu $\mathrm{P}$, et al. Phyto-mediated photo catalysed green synthesis of silver nanoparticles using Durio zibethinus seed extract: Antimicrobial and cytotoxic activity and photocatalytic applications. Molecules 2018;23:3311.

67. Venkatesan J, Kim SK, Shim MS. Antimicrobial, antioxidant, and anticancer activities of biosynthesized silver nanoparticles using marine algae Ecklonia cava. Nanomaterials (Basel) 2016;6:235.

68. Satyavani K, Gurudeeban S, Ramanathan T, Balasubramanian T. Biomedical potential of silver nanoparticles synthesized from calli cells of Citrullus colocynthis (L.) schrad. J Nanobiotechnology 2011;9:43.

69. Mohanta YK, Panda SK, Jayabalan R, Sharma N, Bastia AK, Mohanta TK, et al. Antimicrobial, antioxidant and cytotoxic activity of silver nanoparticles synthesized by leaf extract of Erythrina suberosa (Roxb.). Front Mol Biosci 2017;4:14.

70. Elbagory A, Meyer M, Cupido C, Hussein A. Inhibition of bacteria associated with wound infection by biocompatible green synthesized gold nanoparticles from South African plant extracts. Nanomaterials (Basel) 2017;7:417.

71. Stolarczyk EU, Stolarczyk K, Łaszcz M, Kubiszewski M, Maruszak $\mathrm{W}$, Olejarz W, et al. Synthesis and characterization of genistein conjugated with gold nanoparticles and the study of their cytotoxic properties. Eur J Pharm Sci 2017;96:176-85.

72. Gopinath K, Kumaraguru S, Bhakyaraj K, Mohan S, Venkatesh KS, Esakkirajan M, et al. Green synthesis of silver, gold and silver/gold bimetallic nanoparticles using the Gloriosa superba leaf extract and their antibacterial and antibiofilm activities. Microb Pathog 2016;101:1-11

73. Rodríguez-Luis OE, Hernandez-Delgadill R, Sánchez-Nájera RI, Martínez-Castañón GA, Niño-Martínez N, Navarro MC, et al. Green synthesis of silver nanoparticles and their bactericidal and antimycotic activities against oral microbes. J Nanomater 2016;2016:10.

74. Huo Y, Singh P, Kim YJ, Soshnikova V, Kang J, Markus J, et al. Biological synthesis of gold and silver chloride nanoparticles by Glycyrrhiza uralensis and in vitro applications. Artif Cells Nanomed Biotechnol 2018;46:303-12.

75. Suman TY, Rajasree SR, Jayaseelan C, Mary RR, Gayathri S, Aranganathan L, et al. GC-MS analysis of bioactive components and biosynthesis of silver nanoparticles using Hybanthus enneaspermus at room temperature evaluation of their stability and its larvicidal activity. Environ Sci Pollut Res Int 2016;23:2705-14.

76. Raghavan BS, Kondath S, Anantanarayanan R, Rajaram R. Kaempferol mediated synthesis of gold nanoparticles and their cytotoxic effects on MCF-7 cancer cell line. Process Biochem 2015;50:1966-76.

77. Cheirmadurai K, Biswas S, Murali R, Thanikaivelan P. Green synthesis of copper nanoparticles and conducting nanobiocomposites using plant and animal sources. RSC Adv 2014;4:19507-11.

78. Ajitha B, Reddy YA, Shameer S, Rajesh KM, Suneetha Y, Reddy PS, et al. Lantana camara leaf extract mediated silver nanoparticles: Antibacterial, green catalyst. J Photochem Photobiol B 2015;149:84-92.

79. Du J, Zhou Z, Zhang X, Wu S, Xiong J, Wang W, et al. Biosynthesis of gold nanoparticles by flavonoids from Lilium casa blanca. J Cluster Sci 2017;28:3149-58.

80. Salgado P, Márquez K, Rubilar O, Contreras D, Vidal G. The effect of phenolic compounds on the green synthesis of iron nanoparticles (FexOy-NPs) with photocatalytic activity. Appl Nanosci 2019;9:371.

81. Kumar HA, Mandal BK, Kumar KM, Maddinedi SB, Sai Kumar T, Madhiyazhagan $\mathrm{P}$, et al. Antimicrobial and antioxidant activities of Mimusops elengi seed extract mediated isotropic silver nanoparticles. Spectrochim Acta A Mol Biomol Spectrosc 2014;130:13-8.

82. Sathishkumar G, Gobinath C, Karpagam K, Hemamalini V, Premkumar K, Sivaramakrishnan S, et al. Phyto-synthesis of silver nanoscale particles using Morinda citrifolia L. And its inhibitory activity against human pathogens. Colloids Surf B Biointerfaces 2012;95:235-40.

83. Eslami S, Ebrahimzadeh MA, Biparva P. Green synthesis of safe zero valent iron nanoparticles by Myrtus communis leaf extract as an effective agent for reducing excessive iron in iron-overloaded mice, a thalassemia model. RSC Adv 2018;8:26144-55.

84. Chahardoli A, Karimi N, Fattahi A. Biosynthesis, characterization, antimicrobial and cytotoxic effects of silver nanoparticles using Nigella arvensis seed extract. Iran J Pharm Res 2017;16:1167-75.

85. Ramteke C, Chakrabarti T, Sarangi BK, Pandey RA. Synthesis of silver nanoparticles from the aqueous extract of leaves of Ocimum sanctum for enhanced antibacterial activity. J Chem 2013;2013:7.

86. Mittal AK, Tripathy D, Choudhary A, Aili PK, Chatterjee A, Singh IP, et al. Bio-synthesis of silver nanoparticles using Potentilla fulgens wall. Ex hook. And its therapeutic evaluation as anticancer and antimicrobial agent. Mater Sci Eng C Mater Biol Appl 2015;53:120-7.

87. Vinodhini A, Govindaraju K, Singaravelu G, Sadiq AM, Kumar VG. Cardioprotective potential of biobased gold nanoparticles. Colloids Surf B Biointerfaces 2014;117:480-6.

88. Satpathy S, Patra A, Ahirwar B, Hussain MD. Antioxidant and anticancer activities of green synthesized silver nanoparticles using aqueous extract of tubers of Pueraria tuberosa. Artif Cells Nanomed Biotechnol 2018;46:S71-85

89. Khan M, Khan M, Adil SF, Tahir MN, Tremel W, Alkhathlan HZ, et al. Green synthesis of silver nanoparticles mediated by Pulicaria glutinosa extract. Int J Nanomedicine 2013;8:1507-16.

90. Sarkar S, Kotteeswaran V. Green synthesis of silver nanoparticles from aqueous leaf extract of Pomegranate (Punica granatum) and their anticancer activity on human cervical cancer cells. Adv Nat Sci Nanosci Nanotechnol 2018;9:25014.

91. Mittal AK, Kumar S, Banerjee UC. Quercetin and gallic acid mediated synthesis of bimetallic (silver and selenium) nanoparticles and their antitumor and antimicrobial potential. J Colloid Interface Sci 2014;431:194-9.

92. Korbekandi H, Chitsazi MR, Asghari G, Bahri Najafi R, Badii A, Iravani $\mathrm{S}$, et al. Green biosynthesis of silver nanoparticles using Quercus brantii (oak) leaves hydroalcoholic extract. Pharm Biol 2015;53:807-12.

93. Tahir K, Ahmad A, Li B, Khan AU, Nazir S, Khan S, et al. Preparation, characterization and an efficient photocatalytic activity of $\mathrm{Au} / \mathrm{TiO} 2$ nanocomposite prepared by green deposition method. Mater Lett 2016;178:56-9.

94. Ghaedi M, Yousefinejad M, Safarpoor M, Khafri HZ, Purkait MK. Rosmarinus officinalis leaf extract mediated green synthesis of silver nanoparticles and investigation of its antimicrobial properties. J Ind Eng Chem 2015;31:167-72.

95. Jadhav K, Deore S, Dhamecha D, Rajeshwari HR, Jagwani S, Jalalpure S, et al. Phytosynthesis of silver nanoparticles: Characterization, biocompatibility studies, and anticancer activity. ACS Biomater Sci Eng 2018;4:892-9.

96. Firoozi S, Jamzad M, Yari M. Biologically synthesized silver nanoparticles by aqueous extract of Satureja intermedia C.A. Mey and the evaluation of total phenolic and flavonoid contents and antioxidant activity. J Nanostruct Chem 2016;6:357

97. Das J, Velusamy P. Catalytic reduction of methylene blue using biogenic gold nanoparticles from Sesbania grandiflora L. J Taiwan Inst Chem Eng 2014;45:2280-5.

98. Abbai R, Mathiyalagan R, Markus J, Kim YJ, Wang C, Singh P, et al. Green synthesis of multifunctional silver and gold nanoparticles from the oriental herbal adaptogen: Siberian ginseng. Int J Nanomedicine 2016;11:3131-43

99. Bogireddy NK, Anand KK, Mandal BK. Gold nanoparticles-synthesis by Sterculia acuminata extract and its catalytic efficiency in alleviating different organic dyes. J Mol Liq 2015;211:868-75.

100. Rajathi FA, Arumugam R, Saravanan S, Anantharaman P. Phytofabrication of gold nanoparticles assisted by leaves of Suaeda monoica and its free radical scavenging property. J Photochem Photobiol B 2014;135:75-80.

101. Thakore S, Rathore PS, Jadeja RN, Thounaojam M, Devkar RV. Sunflower oil mediated biomimetic synthesis and cytotoxicity of monodisperse hexagonal silver nanoparticles. Mater Sci Eng C Mater Biol Appl 2014;44:209-15.

102. Prasad R, Swamy SV. Antibacterial activity of silver nanoparticles synthesized by bark extract of Syzygium cumini. J Nanopart 2013;2013:431218.

103. Kumar V, Yadav SC, Yadav SK. Syzygium cumini leaf and seed extract mediated biosynthesis of silver nanoparticles and their characterization. J Chem Technol Biotechnol 2010;85:1301-9.

104. Thampi N, Shalini JV. Bio-prospecting the in vitro antioxidant and anticancer activities of silver nanoparticles synthesized from the leaves of Syzyegium samarangense. Int J Pharm Pharm Sci 2015;7:269-74.

105. Correa SN, Naranjo AM, Herrera AP. Biosynthesis and characterization of gold nanoparticles using extracts of Tamarindus indica L leaves. J Phys Conf Ser 2016;687:12082.

106. Alegria EC, Ribeiro AP, Mendes M, Ferraria AM, do Rego AM, Pombeiro AJ, et al. Effect of phenolic compounds on the synthesis of gold nanoparticles and its catalytic activity in the reduction of nitro 
compounds. Nanomaterials (Basel) 2018;8:320.

107. Rajaram K, Aiswarya DC, Sureshkumar P. Green synthesis of silver nanoparticle using Tephrosia tinctoria and its antidiabetic activity. Mater Lett 2015;138:251-4.

108. Gopinath K, Gowri S, Karthika V, Arumugam A. Green synthesis of gold nanoparticles from fruit extract of Terminalia arjuna, for the enhanced seed germination activity of Gloriosa superba. J Nanostruct Chem 2014;4:115.

109. Ankamwar B. Biosynthesis of gold nanoparticles (green-gold) using leaf extract of Terminalia catappa. E J Chem 2010;7:1334-9.

110. Khorrami S, Zarrabi A, Khaleghi M, Danaei M, Mozafari MR. Selective cytotoxicity of green synthesized silver nanoparticles against the MCF-7 tumor cell line and their enhanced antioxidant and antimicrobial properties. Int J Nanomedicine 2018;13:8013-24

111. Velmurugan P, Anbalagan K, Manosathyadevan M, Lee KJ, Cho M,
Lee SM, et al. Green synthesis of silver and gold nanoparticles using Zingiber officinale root extract and antibacterial activity of silver nanoparticles against food pathogens. Bioprocess Biosyst Eng 2014;37:1935-43.

112. Halder A, Das S, Bera T, Mukherjee A. Rapid synthesis for monodispersed gold nanoparticles in kaempferol and antileishmanial efficacy against wild and drug resistant strains. RSC Adv 2017;7:14159-67.

113. Nazeruddin GM, Prasad NR, Prasad SR, Shaikh YI, Waghmare SR, Adhyapak P. Coriandrum sativum seed extract assisted in situ green synthesis of silver nanoparticle and its anti-microbial activity. Ind Crops Prod 2014;60:212-6.

114. Dash SS, Bag BG. Synthesis of gold nanoparticles using renewable Punica granatum juice and study of its catalytic activity. Appl Nanosci 2014;4:55-9. 